\title{
Effect of interfacial serum proteins on melanoma cell adhesion to biodegradable poly(L-lactic acid) microspheres coated with hydroxyapatite.
}

\section{$\operatorname{AUTHOR}(\mathrm{S})$ :}

Shinto, Hiroyuki; Hirata, Takuya; Fukasawa, Tomonori; Fujii, Syuji; Maeda, Hayata; Okada, Masahiro; Nakamura, Yoshinobu; Furuzono, Tsutomu

\section{CITATION:}

Shinto, Hiroyuki ... [et al]. Effect of interfacial serum proteins on melanoma cell adhesion to biodegradable poly(L-lactic acid) microspheres coated with hydroxyapatite.. Colloids and surfaces. B, Biointerfaces 2013, 108: 8-15

\section{ISSUE DATE:}

2013-02

URL:

http://hdl.handle.net/2433/173348

\section{RIGHT:}

C 2013 Elsevier B.V.; この論文は出版社版でありません。引用の際には 出版社版をご確認ご利用ください。; This is not the published version. Please cite only the published version. 


\section{Effect of interfacial serum proteins on melanoma cell}

\section{adhesion to biodegradable poly(L-lactic acid) \\ microspheres coated with hydroxyapatite}

Hiroyuki Shinto, ${ }^{*} 1$ Takuya Hirata, ${ }^{1}$ Tomonori Fukasawa, ${ }^{1}$ Syuji Fujii, ${ }^{2}$ Hayata Maeda, ${ }^{2}$ Masahiro Okada, ${ }^{3}$ Yoshinobu Nakamura, ${ }^{2}$ and Tsutomu Furuzono ${ }^{4}$

${ }^{1}$ Department of Chemical Engineering, Kyoto University, Katsura, Nishikyo-ku, Kyoto 615-8510, Japan

${ }^{2}$ Department of Applied Chemistry, Faculty of Engineering, Osaka Institute of Technology, 5-16-1 Ohmiya, Asahi-ku, Osaka 535-8585, Japan

${ }^{3}$ Department of Biomaterials, Osaka Dental University, 8-1 Kuzuha-Hanazono, Hirakata, Osaka 5731121, Japan

${ }^{4}$ Department of Biomedical Engineering, School of Biology-Oriented Science and Technology, Kinki University, 930 Nishi-Mitani, Kinokawa, Wakayama 649-6493, Japan

* Author to whom correspondence should be addressed.

Phone: +81-75-383-2671, Fax: +81-75-383-2651, e-mail: shinto@ cheme.kyoto-u.ac.jp 


\section{Abstract}

We have measured the interaction forces between a murine melanoma cell and a poly(L-lactic acid) (PLLA) microsphere coated with/without hydroxyapatite (HAp) nanoparticles (i.e., an HAp/PLLA or a bare PLLA microsphere) in a serum-free culture medium, using atomic force microscopy (AFM) with colloid probe technique, in order to investigate how the HAp-nanoparticle coating as well as interfacial serum proteins influence the cell-microsphere adhesion. The cell adhesion force of the HAp/PLLA microspheres was 1.4-fold stronger than that of the bare PLLA microspheres. When the microspheres were pretreated with a culture medium supplemented with $10 \%$ fetal bovine serum, the cell adhesion force of the HAp/PLLA microspheres was increased by a factor of 2.1; in contrast, no change was observed in the cell adhesion force of the bare PLLA microspheres before/after the pretreatment. Indeed, the cell adhesion force of the HAp/PLLA was 2.8-fold larger than that of the bare PLLA after the pretreatment. Additionally, we have investigated the effect of interfacial serum proteins on the zeta potentials of these microspheres. On the basis of the obtained results, possible mechanism of cell adhesion to the HAp/PLLA and bare PLLA microspheres in the presence/absence of the interfacial serum proteins is discussed.

\section{Keywords:}

Atomic force microscopy; Cell adhesion; Hydroxyapatite; Poly(L-lactic acid); Core-shell microspheres. 


\section{Introduction}

Toward efficient development of biomaterials with higher biocompatibility, it is of critical importance to understand and control the physicochemical and biological interactions that occur at the interface between materials and tissues (or cells). Especially, adhesion of mammalian cells to materials plays a key role in many processes within multicellular organisms; these processes include the formation and the cohesion of tissues, cell differentiation, cell motility, and pathologies such as cancer proliferation and metastasis. In order to understand the mechanism of adhesion between a mammalian cell and a material surface, one should often consider the presence of proteins adsorbed on the material surface; particularly, extracellular matrix (ECM) proteins such as fibronectin, vitronectin, and laminin play a crucial role in the cell-material adhesion [1]. The integrins are transmembrane adhesion molecules and act as cell-adhesion receptors that form contacts with ECM. In a culture medium containing ECM proteins, the mammalian cells interact with material surfaces via the ECM proteins adsorbed thereon, where the integrins in cell membranes bind to the ECM proteins [2,3]. Indeed, in cell culture systems, a serum including ECM proteins is usually added to the culture medium, helping anchorage-dependent cells adhere to the surfaces of culture dishes [4].

Atomic force microscopy (AFM) is a good tool to measure the interaction forces of a living cell with a substrate and has been widely employed for cell adhesion studies [5]. Thie et al. [6] are the first to report the AFM study of the interaction forces between a living mammalian cell and a substrate. Since then, lots of research groups have reported the AFM studies of cell adhesion. Their main focus is on measuring and interpreting at a molecular level the specific interactions for a pair of the ligands and receptors related to the cell adhesion, where the ligands are fixed at the substrates or the AFM probes [7]. Despite their importance for understanding the cell-material adhesion, only a few AFM studies have reported the mammalian cell adhesion to the artificial materials of the metallic surfaces [8] and the simple surface chemistries such as charged hydrophilic (e.g., carboxyl and amino) groups, uncharged 
hydrophilic (e.g., hydroxyl and polyethylene glycol) groups, hydrophobic (e.g., alkyl and phenyl) groups [8-17].

Hydroxyapatite (HAp) is the main mineral of bones and teeth, and artificially synthesized HAp has been extensively used in a variety of applications (such as biomaterials, ion exchangers, adsorbents, and catalysts) by exploiting their biocompatibility and adsorbability with many compounds [18-20]. It has been revealed that nanostructured HAp has better biocompatibility than conventional ones [21-24]. Recently, biodegradable microspheres coated with HAp nanoparticles were synthesized via Pickering emulsion method: oil droplets, which dissolved biodegradable polymers therein and were covered with HAp nanoparticles, were stably dispersed in an aqueous medium and the solvent oil therein was then evaporated [25]. In the Pickering emulsion method, the HAp nanoparticles were employed not only as a particulate emulsifier to prevent flocculation of the emulsion droplets and the obtained microspheres, but also as an enhancer for cell adhesion properties of the microspheres. This method has an advantage: neither molecular surfactant nor polymeric stabilizer was used, which is usually used to fabricate/stabilize the microspheres in liquid media and involves potential risk to cause allergy-like reactions and carcinogenicity [26,27]. Fujii et al. [25] showed that the HAp nanoparticles on the surface of the poly(L-lactic acid) (PLLA) microspheres promote the adhesion and spreading of L929 fibroblast cells thereon, where the cells suspended in a culture medium supplemented with $10 \%$ fetal bovine serum were seeded on the HAp nanoparticle-coated PLLA (HAp/PLLA) microspheres fixed on substrates and were incubated for $24 \mathrm{~h}$. Furthermore, Mima et al. [28] confirmed that the HAp/PLLA microspheres can work as an effective injectable scaffold, which enhances cell-based therapeutic angiogenesis with bone marrow mononuclear cells (BMNCs): when the BMNCs derived from transgenic mice expressing enhanced green fluorescent protein (GFP) were injected into an ischemic muscle, the muscle GFP level in the case of co-injection with the HAp/PLLA microspheres was about 5-fold higher than that in the case of BMNCs alone or co-injection with the bare PLLA microspheres within 7 days after injection. Here, Mima et al. [28] employed a serum-free culture medium to suspend the BMNCs and the microspheres therein. Although these previous studies indicate that the HAp/PLLA 
microspheres have affinity for cells higher than the bare PLLA microspheres, the interactions of the microspheres with cells are not understood well. The aim of the present study is to better understand the cell adhesion to the HAp/PLLA and bare PLLA microspheres and the effects of serum proteins on the cell-microsphere adhesion.

In the present study, we report the direct measurement of the interaction force between a murine melanoma cell and a microsphere of the HAp/PLLA or the bare PLLA in a culture medium, using AFM with colloid probes. We have also investigated the effects of the adsorbed serum proteins on the zeta potentials of these microspheres as well as on the cell-microsphere adhesion, where the microspheres were pretreated with the culture media supplemented with $10 \%$ fetal bovine serum. On the basis of the obtained results, possible mechanism of cell adhesion to the HAp/PLLA and bare PLLA microspheres in the presence/absence of the interfacial serum proteins is discussed.

\section{Materials and methods}

\subsection{Cell line and culture conditions}

The murine melanoma cell line (B16F10; ATCC CRL-6475) was cultured in a complete medium composed of an MEM medium (05900, Eagle's minimum essential medium with kanamycin, without Lglutamine or sodium bicarbonate; Nissui Pharmaceutical, Tokyo, Japan), L-glutamine (Nacalai Tesque, Kyoto, Japan) and 10\% fetal bovine serum (FBS; JRH Biosciences, Lenexa, KS, USA); additionally, sodium bicarbonate (Nacalai Tesque, Kyoto, Japan) was used to adjust the $\mathrm{pH}$ to 7.4. In prior to use, the FBS was heat-inactivated. The anchorage-dependent cells were cultured statically in a $75-\mathrm{cm}^{2}$ flask (3110-075; IWAKI, Tokyo, Japan) containing a 10-mL complete medium, and the flask was stored in an incubator, inside which a moist atmosphere of $5.0 \% \mathrm{CO}_{2}$ was maintained at temperature of $37.0{ }^{\circ} \mathrm{C}$. This ensured that the complete medium maintained the physiological $\mathrm{pH}$ of 7.4. The complete medium 
was changed every 2 days. The cells were subcultured every 4 days, when they formed a subconfluent monolayer (the surface density, $0.7 \times 10^{5}$ cells $/ \mathrm{cm}^{2}$ ): It is noted that a confluent monolayer of the B16F10 cells gave $0.8 \times 10^{5}$ cells $/ \mathrm{cm}^{2}$. Unless specified otherwise, all water used in the experiments was purified using a system of AutoStill and Autopure (WG202 and WR600A; Yamato Scientific, Tokyo, Japan) to give a resistance of $18.2 \mathrm{M} \Omega \cdot \mathrm{cm}$ and a total organic carbon of less than $20 \mathrm{ppb}$.

\subsection{Preparation of cell samples}

To prepare the B16F10 cell samples for AFM measurements, the $75-\mathrm{cm}^{2}$ flask with the subconfluent monolayer cells prepared as explained in Section 2.1 was first rinsed with $10 \mathrm{~mL}$ of Dulbecco's phosphate-buffered saline without calcium or magnesium (DPBS, 21600; GIBCO, Grand Island, NY, USA) after removal of the complete medium (10\% FBS-supplemented MEM); subsequently, the cells were separated from the flask surface by trypsinization with a $1-\mathrm{mL}$ DPBS solution of $0.25 \%$ trypsin and $0.02 \%$ EDTA. A fresh 9-mL complete medium was then added into the flask, giving a 10-mL cell suspension of $5 \times 10^{5}$ cells $/ \mathrm{mL}$. Proper amounts of this cell suspension in the complete medium were filled in fresh $\phi 35$-mm polystyrene dishes (3000-035; IWAKI) to satisfy a liquid surface height of $2 \mathrm{~mm}$ and different cell concentrations of $1 \times 10^{5}, 3 \times 10^{5}$, and $5 \times 10^{5}$ cells $/ \mathrm{mL}$. For subculture, $0.25 \mathrm{~mL}$ of the cell suspension was added into a fresh $75-\mathrm{cm}^{2}$ flask including a fresh $10-\mathrm{mL}$ complete medium, giving a cell concentration of $2 \times 10^{3}$ cells $/ \mathrm{cm}^{2}$.

After 1-day incubation, all the $\phi 35$-mm dishes were checked by a light microscope; consequently, only the culture dishes presenting the subconfluent monolayer cells of $80 \%$ surface occupation were selected for AFM measurements. The selected culture dishes were rinsed with 2-mL DPBS after removal of the complete medium therein, and filled with $2.5 \mathrm{~mL}$ of a Dulbecco's modified Eagle medium (DMEM high glucose including 25.03-mM HEPES buffer, 21063-029; GIBCO) so that the pH of the solution therein was maintained at 7.4 even in the outside environment for several hours. FBS was never added to the DMEM solution for the cell samples. 


\subsection{Scanning electron microscopy (SEM) observation of cell surfaces}

The B16F10 cells were grown on 9-mm $\times$ 9-mm coverslips (Matsunami, Osaka, Japan) being sunk into the $\phi 35-\mathrm{mm}$ polystyrene dishes, where the cells suspended in the complete medium at $2 \times 10^{4}$ cells $/ \mathrm{mL}$ were seeded. Before use, the coverslips were sterilized in ethanol overnight, washed thoroughly in water, and dried in air inside a bio-clean bench. After 1-day incubation, the dishes with the coverslips were rinsed with 2-mL DPBS after removal of the complete medium therein, and filled with 2.5-mL DMEM. The samples were stored in the incubator for $30 \mathrm{~min}$ and then rinsed with 2-mL DPBS after removal of DMEM. For subsequent SEM observation, the samples were fixed with paraformaldehyde, post-fixed with $\mathrm{OsO}_{4}$, and dehydrated in an ethanol series and t-butyl alcohol, followed by being frozen in a small amount of t-butyl alcohol and freeze-dried, as described elsewhere [16]. Finally, the samples were sputtered with a conductive layer of 5-nm thickness gold using an Emitech K575XD (Quorum Technologies, Ashford, UK) and imaged using a Keyence VE-8800 (Osaka, Japan).

\subsection{Fabrication of HAp/PLLA core-shell microspheres}

HAp nanoparticle-coated PLLA (HAp/PLLA) microspheres were fabricated via the Pickering emulsion method in the absence of any molecular surfactants, as described in our previous study [25]. The HAp nanoparticles used as a particulate stabilizer were synthesized via wet chemical process, using the same protocol as described in our early study [29]. A stock aqueous dispersion of the HAp nanoparticles (0.04 wt\% solid content) was prepared by serial dilution. An aliquot of the HAp dispersion $(25 \mathrm{~g})$ were then hand-shaken with a dichloromethane $\left(\mathrm{CH}_{2} \mathrm{Cl}_{2}\right)$ solution of PLLA (total $2.5 \mathrm{~g}$, $1 \mathrm{wt} \%$ solid content) at $25{ }^{\circ} \mathrm{C}$ for $30 \mathrm{~s}$. The HAp/PLLA microspheres were obtained after the evaporation of $\mathrm{CH}_{2} \mathrm{Cl}_{2}$ from the emulsion at $25^{\circ} \mathrm{C}$. Bare PLLA microspheres were prepared by removal 
of the HAp nanoparticles from the HAp/PLLA microspheres using a aqueous solution of nitric acid $\left(\mathrm{HNO}_{3} ; \mathrm{pH} 2.0\right)$, which could dissolve the HAp component. These bare PLLA microspheres were used after washing with water to remove ionic species generated by dissolution of the HAp nanoparticles.

\subsection{Zeta potentials of microspheres, HAp nanoparticles, and cells}

The zeta potentials of the bare PLLA and the HAp/PLLA microspheres as well as the HAp nanoparticles were measured by electrophoresis at $25^{\circ} \mathrm{C}$ using Zetasizer Nano ZS (Malvern Instruments, Worcestershire, UK), where dynamic light scattering of the dispersion in an oscillatory electrical field was measured. After centrifugation of an aliquot of the stock dispersion and removal of the supernatant, the remained particulate dispersoid (i.e., the microspheres or the HAp nanoparticles) was resuspended in either an aqueous solution of $0.1-\mathrm{mM}$ sodium chloride $(\mathrm{NaCl})$ or DMEM. In addition to the nontreated dispersoid, the pretreated dispersoid was prepared as follows. The particulate dispersoid remained after centrifugation and decantation was resuspended in either 1-mL DMEM or 1-mL DMEM supplemented with $10 \%$ FBS in a clean microtube. The microtube was rigorously shaken with a test tube mixer (Se08; TAITEC, Saitama, Japan) for 3 min and constantly rotated with RT-30mini (TAITEC) for 30 min at room temperature in an air-conditioned laboratory $\left(23 \pm 2{ }^{\circ} \mathrm{C}\right)$. The particulate suspension was centrifuged, the supernatant was discarded, and the resulting precipitate was resuspended in $1 \mathrm{~mL}$ of $0.1-\mathrm{mM} \mathrm{NaCl}$ solution by rigorous shaking. This procedure was repeated three times in order to eliminate free and loosely-adsorbed serum proteins as well as free and loosely-adsorbed components of DMEM from the dispersion medium of $0.1-\mathrm{mM} \mathrm{NaCl}$ solution. Likewise, the procedure was repeated three times before a last resuspension in 1-mL DMEM by rigorous shaking, whereby nonadsorbed serum proteins were eliminated from the dispersion medium of DMEM. The nontreated/pretreated particulate dispersoids suspended in $0.1-\mathrm{mM} \mathrm{NaCl}$ solution and DMEM were placed in a disposable capillary cell (DTS1061; Malvern Instruments) and a Universal Dip Cell (ZEN1002; Malvern Instruments), respectively, and the measurement of every sample was carried out at $25^{\circ} \mathrm{C}$ in triplicate. 
The surface charge of the microspheres was calculated from the mean electrophoretic mobility using Smoluchowski's equation, where the viscosity and dielectric constant of media were used as calculation parameters.

For comparison with the bare PLLA microspheres, we employed the carboxyl-modified polystyrene (PS-COOH) microspheres, which had a diameter of $9.95 \pm 0.53 \mu \mathrm{m}$ and a carboxyl-group density of 1.06 groups $/ \mathrm{nm}^{2}$ (Bangs Laboratories, Fishers, IN, USA). Before use, the PS-COOH microspheres were washed three times with water by a series of centrifugation, decantation, and resuspension. The zeta potentials of the nontreated/pretreated PS-COOH microspheres were measured in the same way as described above.

In addition, we measured the zeta potentials of the B16F10 cells suspended in 100-mM NaCl solution. Before the measurements, we gently washed the cells two times with $100-\mathrm{mM} \mathrm{NaCl}$ solution by a series of centrifugation, decantation, and resuspension in order to remove the trypsin and serum proteins from the medium of the cell suspensions.

\subsection{Preparation of colloid probes}

An AFM probe (Model NP; Veeco Instruments, Santa Barbara, CA, USA), which has a V-shaped 200- $\mu \mathrm{m}$ long cantilever (a spring constant, $0.06 \mathrm{~N} / \mathrm{m}$ ) with an $\mathrm{Si}_{3} \mathrm{~N}_{4}$ pyramidal tip on its end, was used. Because the microspheres were dispersed in water, the microsphere suspension in a clean microtube was placed in a vacuum chamber for $3 \mathrm{~h}$ to remove the water. A single microsphere of $8-10 \mu \mathrm{m}$ diameter was selectively glued to the end of the cantilever with a very small amount of epoxy adhesive (Araldite Standard; Huntsman Japan, Kobe), using an XYZ micromanipulator system (MMO-220A, MMO-202ND, and MN-4; NARISHIGE, Tokyo, Japan) mounted on a light microscope (LV100D-U; Nikon, Tokyo, Japan). This colloid probe was left in a clean box at room temperature over $12 \mathrm{~h}$, which allowed the epoxy adhesive to harden sufficiently. The colloid probes prepared are shown in Fig. 1. 


\subsection{AFM measurement of cell-microsphere interaction forces}

Our methods for AFM measurements were explained elsewhere [16]. An MFP-3D-BIO AFM (Asylum Research, Santa Barbara, CA, USA) combined with an inverted light microscope (TE2000-U; Nikon, Tokyo, Japan) placed on an active vibration isolation system (TS-140; Herz, Yokohama, Japan) was used to measure the interaction forces between a living cell and a colloid probe in a serum-free culture medium of DMEM at room temperature in an air-conditioned laboratory $\left(23 \pm 2{ }^{\circ} \mathrm{C}\right)$. The cantilever with the colloid probe was fixed on the AFM head (the Z-scanner). In prior to the cellular force measurement, the optical lever sensitivity was determined using a clean mica sheet which was sunk in a fresh $\phi 35-\mathrm{mm}$ dish containing water, whereby the surface cleanliness of the colloid probe was checked at the same time. When the effect of serum proteins on the cell-microsphere adhesion was investigated, the colloid probe was then immersed in DMEM supplemented with 10\% FBS filling another fresh dish for $30 \mathrm{~min}$ at room temperature, which allowed some of the serum proteins to adsorb on the surface of the colloid probe. In replacement of these dishes, the cell culture dish prepared as in Section 2.2 was then placed on the AFM base (the XY-scanner equipped with the manual XYtranslation stage), below which the light microscope was positioned; this enabled us not only to locate the colloid probe over the nucleus of a living cell, but also to monitor the cell during the force measurement. Because the living anchorage-dependent cells during interphase adhered to the dish surface and flatten or spread out thereon, they could be easily distinguished from the mitosis-phase cells and the dead cells, which evidently rounded up to exhibit a spherical shape and were much more loosely attached to the surface [4]. The force measurement was started using the colloid probe and the cells in the culture dish.

In measurement of a compression force curve, the colloid probe was brought in contact with the cell of interest at a speed of $0.8 \mu \mathrm{m} / \mathrm{s}$ and a minimum indentation depth of about $1 \mu \mathrm{m}$ required for the probe to reach the cell surface and to give a compliance region. This Z-scan speed was confirmed to be low enough to reduce or eliminate the hydrodynamic effects in the compression/decompression force curves, 
by comparison among the force curves obtained using higher and lower scan speeds. Once the indentation depth reached the typical values of $1.3 \pm 0.3 \mu \mathrm{m}$, resulting in the loading forces of $0.6-1.8$ $\mathrm{nN}$, the colloid probe was then allowed to reside on the cell surface for $5 \mathrm{~min}$, during which time the cell did not migrate but deformed due to the contact with the probe. After this predefined dwell time of 5 min, the probe was moved away from the cell surface. In the case of strong adhesions between the cell and the probe, they did not always separate completely after the force cycle of compression, residence, and decompression. For this reason, the probe was moved to another place after the force cycle, and then immediately returned to its original position, in order to break any remaining bonds between the probe and cell; thereafter, another compression force curve was then collected, the baseline of which was used to define the zero force position for the decompression force curve [11-17]. Next, the cell culture dish was moved using the manual XY-translation stage of the AFM base and the next force cycle was then started over the nucleus of another cell of interest. By repeating this procedure, typically 10-15 force curves were obtained using a pair of the colloid probe and cell culture dish. Every force curve was obtained at the position over the nucleus of different cells $(n=48-188)$, as summarized in Table 1.

\subsection{Data analysis}

The collected data of the zeta potentials were expressed as the mean \pm standard deviation (s.d.). The force-displacement curves obtained from AFM were analyzed using IGOR Pro software, with which the MFP-3D-BIO AFM system was equipped. The significance of the differences among multiple independent setups was tested by the Mann-Whitney U-test or the Steel-Dwass test, using Microsoft Excel 2003 software and its add-in of Ekuseru-Toukei 2010 (Social Survey Research Information, Tokyo, Japan). $P \leq 0.05$ was considered statistically significant. 


\section{Results and discussion}

\subsection{Zeta potentials of microspheres}

Table 1 shows the zeta potentials of the bare PLLA and the HAp/PLLA microspheres before/after 30min pretreatment with a serum-free DMEM or a 10\% FBS-supplemented DMEM. The bare PLLA microspheres before pretreatment exhibited the zeta potential of $-77 \pm 3 \mathrm{mV}$ in a $0.1-\mathrm{mM} \mathrm{NaCl}$ aqueous solution. This large value of negative charge originated from the significant number of ester, carboxyl $(-\mathrm{COOH})$, hydroxyl $(-\mathrm{OH})$ groups existing at the surface of the bare PLLA microspheres: in general, the ester linkage of PLLA would break on hydrolysis to expose carboxyl and hydroxyl groups on the surface. After the pretreatment with the serum-free DMEM, the negative surface charge reduced in magnitude to $-57 \pm 7 \mathrm{mV}$, indicating that some components of DMEM (e.g., amino acids, vitamins, and glucose) adsorbed onto the bare PLLA microsphere surface to mask in part the negatively charged surface. The pretreatment with the FBS-supplemented DMEM further lessened the magnitude of the zeta potential down to $-48 \pm 5 \mathrm{mV}$, suggesting that some basic serum proteins rather than acidic serum proteins adsorbed onto the bare PLLA microsphere surface to mask further the negatively charged surface. Similar effects of these two types of pretreatments on zeta potentials were observed for the PS$\mathrm{COOH}$ microspheres, as listed in Table 1.

The HAp/PLLA microspheres before pretreatment exhibited the zeta potential of $-19 \pm 2 \mathrm{mV}$, which was one-forth of the bare PLLA microspheres. This big difference of $58 \mathrm{mV}$ in zeta potential between these two types of microspheres was attributed to the HAp nanoparticles that had the zeta potential of $-4 \pm 1 \mathrm{mV}$ and covered the negatively charged surface of PLLA microspheres (compare parts a and $\mathrm{b}$ of Fig. 1). Indeed, the HAp nanoparticles $\left[\mathrm{Ca}_{10}\left(\mathrm{PO}_{4}\right)_{6}(\mathrm{OH})_{2}\right]$ exhibited the $c$ planes with positively charged $\mathrm{Ca}^{2+}$ sites as well as the $a$ planes with negatively charged $\mathrm{PO}_{4}{ }^{3-}$ sites [30]. After the pretreatment with the serum-free DMEM, the HAp/PLLA microspheres exhibited the surface potential of $-30 \pm 5 \mathrm{mV}$, 
indicating that some components of DMEM adsorbed onto the HAp/PLLA microsphere surface to increase the net negative charge of the surface. The pretreatment with the $10 \%$ FBS-supplemented DMEM further increased the magnitude of the zeta potential up to $-38 \pm 2 \mathrm{mV}$, suggesting that some acidic serum proteins rather than basic serum proteins adsorbed onto the HAp/PLLA microsphere surface to increase further the net negative charge of the surface.

Thus, the effects of these two types of pretreatments on the zeta potentials of the HAp/PLLA microspheres were diametrically opposite to those for the bare PLLA microspheres. The pretreatments by the serum-free and the $10 \%$ FBS-supplemented culture media reduced the difference in zeta potential between these two types of microspheres from $58 \mathrm{mV}$ to 27 and $10 \mathrm{mV}$, respectively. Our results are in agreement with the results reported in the earlier studies [31,32], indicating that the protein adsorption on the surface of particles affects their surface charge.

\subsection{Morphology of apical surface of cells}

SEM was used to illustrate the surface of the B16F10 cells and the micrographs are shown in Fig. 2. The apical surface of the cells appeared to be rather rough and exhibited a dense lawn of microvilli and microridges. The complicated structure of the cell surface explains one of the reasons why molecularlevel interpretation of the force-displacement curves is very difficult, as will be mentioned in Section 3.3 .

\subsection{Force-displacement curves of a microsphere interacting with a cell}

Figure 3a illustrates a cycle of the force measurement by AFM. Fig. 3b displays typical force-displacement curves during compression and decompression measured between the living B16F10 cell and HAp/PLLA microsphere of $10-\mu \mathrm{m}$ diameter in the serum-free culture medium, where the microsphere was pretreated with the $10 \%$ FBS-supplemented DMEM. The compression curve 
displayed zero force at the distances longer than $\sim 1 \mu \mathrm{m}$, at which a repulsive force was detected. This repulsion at the distances shorter than $\sim 1 \mu \mathrm{m}$ was not electrostatic in origin [16], but probably originated from both steric stabilization forces and viscoelastic forces; the former are caused by the compression of a dense lawn of microvilli on the cell surface (see Fig. 2) as well as a hydrated layer of long-chain polymer molecules (proteins and carbohydrates) thereon, while the latter result from the viscoelastic property of a cell [33]. During the dwell time of 5 min, no significant change was observed in the force-displacement curve and the microsphere was not likely to enter the B16F10 cell. Similar results were obtained for the other condition and/or type of PLLA microspheres (see Supplementary data, Figs. S1 and S2). Thus, the B16F10 cells never took up the bare PLLA and the HAp/PLLA microspheres of $8-10-\mu \mathrm{m}$ diameter during the time period of $5 \mathrm{~min}$, regardless of whether the microspheres were pretreated with serum-supplemented culture media or not. One can roughly estimate the contact area of a colloid probe with the cell surface as $S_{0}=2 \pi R^{2}\left[1-(1-d / R)^{1 / 2}\right]$ from the compression force curve with the Hertz contact model $[9,16]$, where a spherical indenter of radius $R$ is considered to be pushed onto a smooth, homogeneous, semi-infinite elastic solid by the indentation depth of $d$. We obtained $S_{0} \sim 20 \mu \mathrm{m}^{2}$, where $R=4-5 \mu \mathrm{m}$ and $d=1.3 \pm 0.3 \mu \mathrm{m}$ were used.

As shown in Fig. 3, a force curve during decompression after compression and residence is the result of detachment of a microsphere from the apical surface of a cell. The decompression force curve for every microsphere showed an initial, large de-adhesion peak followed by several small steps of 20-40 pN (see Supplementary data, Figs. S1 and S2), indicating that two types of the microspheres employed in the present study adhered onto the apical surface of the B16F10 cell shown in Fig. 2. The small steps are attributable to the breaking of the multiple bonds formed at different locations of close contact between the cell and microsphere surfaces. Similar results of the decompression force curves were obtained for the other pretreatment and/or type of PLLA microspheres (see Supplementary data, Figs. $\mathrm{S} 1$ and S2). It is still challenging to interpret the decompression force curve between the cell and material surfaces from a molecular point of view, because a large number of known/unknown adhesion processes can occur simultaneously [7] and the apical surface of cells often exhibits a rather rough and 
complex structure as shown in Fig. 2. For this reason, the magnitude of the attractive force at the initial de-adhesion peak $\left(F_{\text {adh }}\right)$ will be used as a measure of the overall cell-microsphere adhesion force in Sections 3.4 and 3.5 .

\subsection{Adhesion forces of a nontreated microsphere onto a cell}

The plots of the distributions of the adhesion force between a living B16F10 cell and a microsphere of bare PLLA or HAp/PLLA in a serum-free DMEM are displayed in Fig. 4 . It is noted that $F_{\text {adh }}$ is the force required for detaching the microsphere from the cell; the larger value of $F_{\text {adh }}$ would indicate the stronger adhesion [7,34]. Every distribution of $F_{\text {adh }}$ was rather broad and scattered, as seen from the interquartile range and the whisker range. The distribution width for $F_{\text {adh }}$ seen from the interquartile range as well as the whisker range can be thought to depict the variation in the surface properties of individual cells, that is, the cell-to-cell difference in the number of sites on the individual cell surface that can bind with the microsphere surface.

Before pretreatment, the values of $F_{\text {adh }}$ for the HAp/PLLA microspheres were significantly larger than those for the bare PLLA microspheres $\left(P<10^{-5}\right)$ : the median for the former is 1.4 -fold larger than that for the latter. This result is quite understandable on the basis of electrostatic interaction between the microsphere surface and the cell surface that was negatively charged, as in Table 1, probably due to the presence of sialic acids and other negatively charged molecules. The bare PLLA microspheres immersed in DMEM were negatively charged to exhibit a net surface charge of $-22 \pm 1 \mathrm{mV}$, while the HAp/PLLA microspheres were even less charged and the net surface charge $(\approx-4 \mathrm{mV})$ was about onefifth of that of the bare PLLA microspheres, as shown in Table 1; particularly, the surface of the HAp/PLLA microspheres should have the positively charged portions (i.e., $\mathrm{Ca}^{2+}$ sites) as well as the negatively charged portions (i.e., $\mathrm{PO}_{4}{ }^{3-}$ sites) [30], whereas that of the bare PLLA microspheres is considered to have negatively charged sites (e.g., carboxyl and hydroxyl groups) other than positive sites. For these reasons, the negatively charged surface of B16F10 cells was electrostatically attracted to 
the surface of the HAp/PLLA microspheres rather than that of the bare PLLA microspheres. In addition, the surface roughness of the HAp/PLLA microspheres increases the specific surface area, leading to the increase in net area of close contact between the rough, hard surface of an HAp/PLLA microsphere (see Fig. 1b,c) and the rough, soft surface of a B16F10 cell (see Fig. 2).

The distribution of $F_{\text {adh }}$ for the bare PLLA microspheres of $8-10-\mu \mathrm{m}$ diameter was statistically the same as that for the PS-COOH microspheres of $9.95-\mu \mathrm{m}$ diameter $(P=0.33>0.05$; see also Table 1$)$ [16], which had the zeta potential of $\approx-5 \mathrm{mV}$ in DMEM. This could be explained by a similarity in surface chemistry between the bare PLLA and the PS-COOH microspheres: these two types of microspheres had a significant number of carboxyl groups on their surfaces. The malignant melanoma cells, such as B16F10 cell used in our studies, remarkably express several types of adhesion receptors from the integrin family of heterodimers of $\alpha$ and $\beta$ subunits [35]. Our previous study indicated that the PS-COOH microspheres interact rather strongly with the surface of B16F10 cell via the nonspecific $\mathrm{COOH}-$ integrin interaction, whereas the unmodified hydrophobic PS (bare PS) microspheres interact weakly with the cell surfaces through van der Waals forces and hydrophobic forces [16]. Consequently, the affinity of these four types of microspheres for the cell surface is expected as (HAp/PLLA) > (bare $\mathrm{PLLA}) \approx(\mathrm{PS}-\mathrm{COOH})>($ bare PS $)$, unless they are pretreated with serum-supplemented culture media.

\subsection{Adhesion forces of a pretreated microsphere onto a cell}

Here, let us consider the effect of serum proteins on the cell-microsphere adhesion. For this purpose, a microsphere glued to the end of an AFM cantilever was pretreated with the $10 \%$ FBS-supplemented DMEM for $30 \mathrm{~min}$, and then the interaction forces of the microsphere with a cell were measured, as explained in Section 2.7. Figure 4 demonstrates that the values of $F_{\text {adh }}$ for the HAp/PLLA were significantly enhanced by the pretreatment $\left(P<10^{-7}\right)$ and the median of $F_{\text {adh }}$ increased by a factor of 2.1 , while no change was observed in the distributions of $F_{\text {adh }}$ for the bare PLLA before/after the 
pretreatment $(P=0.99)$. Indeed, the median of $F_{\text {adh }}$ for the HAp/PLLA was 2.8-fold larger than that for the bare PLLA after the pretreatment $\left(P<10^{-7}\right)$. This result is not explained by the zeta potentials, where the bare PLLA and the HAp/PLLA microspheres coated with serum proteins had almost the same zeta potentials of about $-12 \mathrm{mV}$ in DMEM, as shown in Table 1.

In order to understand the mechanism of adhesion between a mammalian cell and a material surface, one should consider the presence of ECM proteins adsorbed on the material surface, as mentioned in Introduction. According to the earlier study of Woo et al. [36], the HAp/PLLA composite scaffolds adsorb more than 1.4 times of total serum protein and much greater amounts of serum fibronectin and vitronectin than the pure PLLA scaffolds, where these scaffolds were incubated in a phosphate-buffered saline containing $10 \%$ FBS for $4 \mathrm{~h}$. If this is the case for the HAp/PLLA and the bare PLLA microspheres used in the present study, one can straightforward understand the reason why the pretreatment of the microspheres with the 10\% FBS-supplemented DMEM enhanced the adhesion of the cell to the HAp/PLLA rather than bare PLLA. It should be noted that in addition to the amount of proteins adsorbed on the microspheres, the conformation of the adsorbed proteins on the bare PLLA and the HAp/PLLA microspheres could be different [36]. As observed by Dolatshahi-Pirouz et al. [37], individual fibronectin molecules tend to attach to HAp nanocrystals in unfolded conformations and the cell-binding domain of the adsorbed fibronectin is readily available, where such cell-recognizing abilities are more pronounced at low fibronectin surface coverage.

\section{Conclusions}

We have investigated the adhesion of a single microsphere of the bare PLLA or the HAp/PLLA onto the apical surface of a single B16F10 cell in a serum-free culture medium and the effects of the adsorbed serum proteins on this microsphere-cell adhesion, using colloid-probe AFM. Before pretreatment of the microspheres, the cell adhesion force of the HAp/PLLA microspheres was 1.4-fold 
stronger than that of the bare PLLA microspheres. This is mainly because the negatively charged surface of B16F10 cells at a physiological $\mathrm{pH}$ was electrostatically attracted to the surface of the HAp/PLLA microspheres rather than that of the bare PLLA microspheres: the former has the positively charged portions (i.e., $\mathrm{Ca}^{2+}$ sites) as well as the negatively charged portions (i.e., $\mathrm{PO}_{4}{ }^{3-}$ sites) [30], whereas the latter has negatively charged sites (e.g., carboxyl and hydroxyl groups) only. In addition, the surface roughness of the HAp/PLLA microspheres increases the specific surface area, leading to the increase in net area of close contact between the microsphere and cell surfaces. When the microspheres were pretreated with a 10\% FBS-supplemented culture medium, the cell adhesion force of the HAp/PLLA microspheres was increased by a factor of 2.1 ; in contrast, no change was observed in the cell adhesion force of the bare PLLA microspheres before/after the pretreatment. Indeed, the adhesion force of the HAp/PLLA was 2.8-fold larger than that of the bare PLLA after the pretreatment. This result is not explained by the electrostatic interactions, where the bare PLLA and the HAp/PLLA microspheres coated with serum proteins had almost the same zeta potentials in the serum-free culture medium. The earlier study demonstrated that HAp/PLLA composite scaffolds adsorb much greater amounts of serum fibronectin and vitronectin than the pure PLLA scaffolds [36]. Assuming that this is the case for the HAp/PLLA and the bare PLLA microspheres used in the present study, the aforesaid result is explained by the fact that a material with greater amounts of ECM proteins (e.g., fibronectin and vitronectin) adsorbed thereon exhibits the higher affinity for mammalian cells via specific ECM-integrin interactions.

\section{Acknowledgements}

H.S. thanks for financial support by the Ministry of Education, Culture, Sports, Science and Technology (MEXT) in Japan (Grants-in-Aid for Scientific Research, No. 22686072/23656489; Strategic Development of Research Infrastructure for Private Universities, No. S1201010), the Japan 
Society for the Promotion of Science (JSPS; Core-to-Core Program, No. 18004), and the Information Center of Particle Technology, Japan. 


\section{Figure Captions}

Fig. 1. Scanning electron micrographs of colloid probes: (a) a bare PLLA microsphere, (b) an HAp/PLLA microsphere, and (c) the magnification of the central region of the HAp/PLLA microsphere shown in panel $b$.

Fig. 2. (a) Scanning electron micrograph of B16F10 cells grown on a coverslip and (b) the magnification of its central region.

Fig. 3. (a) Illustration of a cycle of the force measurement by AFM: I, start/end; II, contact; III, retract. (b) Force-displacement curve during compression (I $\rightarrow$ II; upper, red colored) and decompression (III $\rightarrow \mathrm{I}$; lower, blue colored) measured between a B16F10 cell and a 10- $\mu \mathrm{m}$ HAp/PLLA microsphere in a serum-free culture medium of DMEM, where the microsphere was pretreated with a $10 \%$ FBSsupplemented DMEM. The dwell time, indentation depth, and Z-scan speed were $5 \mathrm{~min}, 1.3 \pm 0.3 \mu \mathrm{m}$, and $0.8 \mu \mathrm{m} / \mathrm{s}$, respectively. (For interpretation of the references to color in figure legend, the reader is referred to the web version of the article.)

Fig. 4. The box plots of the distributions of the adhesion force between a living B16F10 cell and a microsphere of the bare PLLA or the HAp/PLLA in a serum-free culture medium of DMEM, where the microspheres were nontreated or pretreated with the 10\% FBS-supplemented DMEM. Each box plot shows six-number summaries: the 0.1-fractile, first quartile, median, third quartile, and 0.9-fractile as well as the mean (solid square plot). Significant differences of $P \leq 0.01$ for all the pairs of four groups were found except for a pair of the nontreated bare PLLA and the pretreated bare PLLA. 
Table 1

Zeta potentials of bare PLLA, HAp/PLLA, and PS-COOH microspheres and HAp nanoparticles as well as the medians of adhesion forces $\left(F_{\text {adh }}\right)$ for the microspheres before/after pretreatment

\begin{tabular}{|c|c|c|c|c|c|c|}
\hline \multirow[t]{2}{*}{ Particulate dispersoid } & \multirow[t]{2}{*}{ Medium } & \multicolumn{3}{|c|}{ Zeta potential $(\mathrm{mV})$} & \multicolumn{2}{|c|}{ Median of $F_{\text {adh }}(\mathrm{nN})$} \\
\hline & & Nontreated & $\begin{array}{l}\text { Pretreated with } \\
\text { DMEM }\end{array}$ & $\begin{array}{l}\text { Pretreated with } \\
10 \% \text { FBS- } \\
\text { supplemented } \\
\text { DMEM }\end{array}$ & Nontreated & $\begin{array}{l}\text { Pretreated with } \\
10 \% \text { FBS- } \\
\text { supplemented } \\
\text { DMEM }\end{array}$ \\
\hline \multirow[t]{2}{*}{ Bare PLLA microsphere } & $0.1-\mathrm{mM} \mathrm{NaCl}$ & $-77 \pm 3$ & $-57 \pm 7(+20)^{\mathrm{a}}$ & $-48 \pm 5(+9)^{b}$ & & \\
\hline & DMEM & $-22 \pm 1$ & & $-12 \pm 1$ & $0.53(n=137)$ & $0.56(n=48)$ \\
\hline \multirow[t]{2}{*}{ HAp/PLLA microsphere } & $0.1-\mathrm{mM} \mathrm{NaCl}$ & $-19 \pm 2$ & $-30 \pm 5(-11)^{\mathrm{a}}$ & $-38 \pm 2(-8)^{b}$ & & \\
\hline & DMEM & $\approx-4$ & & $-11 \pm 1$ & $0.73(n=188)$ & $1.54(n=101)$ \\
\hline \multirow[t]{2}{*}{ PS-COOH microsphere } & $0.1-\mathrm{mM} \mathrm{NaCl}$ & $-43 \pm 4$ & $-31 \pm 2(+12)^{\mathrm{a}}$ & $-19 \pm 2(+12)^{b}$ & & \\
\hline & DMEM & $\approx-5$ & & $\approx-3$ & $0.60(n=51)^{\mathrm{c}}$ & \\
\hline HAp nanoparticles & $0.1-\mathrm{mM} \mathrm{NaCl}$ & $-4 \pm 1$ & $-3 \pm 1(+1)^{\mathrm{a}}$ & $-23 \pm 1(-20)^{\mathrm{b}}$ & & \\
\hline B16F10 cells & $100-\mathrm{mM} \mathrm{NaCl}$ & $-14 \pm 2$ & & & & \\
\hline
\end{tabular}

${ }^{\mathrm{a}}$ The value in the parentheses was calculated by subtraction of the zeta potential of the nontreated dispersoid from that of the DMEM-pretreated dispersoid, indicating the contribution of DMEM components adsorbed on the dispersoid surface to the zeta potential.

${ }^{\mathrm{b}}$ The value in the parentheses was calculated by subtraction of the zeta potential of the DMEMpretreated dispersoid from that of the dispersoid pretreated with a 10\% FBS-supplemented DMEM, indicating the contribution of FBS components adsorbed on the dispersoid surface to the zeta potential.

${ }^{\mathrm{c}}$ Data taken from our previous study [16]: the force measurements were carried out in L-15 culture media, whose components and composition were similar to those of DMEM used in the present study. 


\section{References}

[1] M.R. Koller, E.T. Papoutsakis, in: M.A. Hjortso, J.W. Roos (Eds.) Cell Adhesion: Fundamentals and Biotechnological Applications, Marcel Dekker, New York, 1994, Chapter 3.

[2] C.D. Tidwell, S.I. Ertel, B.D. Ratner, B.J. Tarasevich, S. Atre, D.L. Allara, Langmuir, 13 (1997) 3404-3413.

[3] N. Faucheux, R. Schweiss, K. Lützow, C. Werner, T. Groth, Biomaterials, 25 (2004) 2721-2730.

[4] R.I. Freshney, Culture of Animal Cells: A Manual of Basic Technique and Specialized Applications, 6th edn., Wiley-Blackwell, Hoboken, New Jersey, 2010.

[5] C.M. Franz, A. Taubenberger, P.-H. Puech, D.J. Müller, Sci. STKE, 2007 (2007) pl5.

[6] M. Thie, R. Röspel, W. Dettmann, M. Benoit, M. Ludwig, H.E. Gaub, H.-W. Denker, Human Reproduction, 13 (1998) 3211-3219.

[7] J. Helenius, C.-P. Heisenberg, H.E. Gaub, D.J. Müller, J. Cell. Sci., 121 (2008) 1785-1791.

[8] M. Benoit, H.E. Gaub, Cells Tissues Organs, 172 (2002) 174-189.

[9] H. Kim, H. Arakawa, T. Osada, A. Ikai, Colloids Surf. B: Biointerfaces, 25 (2002) 33-43.

[10] H. Kim, H. Arakawa, T. Osada, A. Ikai, Ultramicroscopy, 97 (2003) 359-363.

[11] C.E. McNamee, N. Pyo, S. Tanaka, I.U. Vakarelski, Y. Kanda, K. Higashitani, Colloids Surf. B: Biointerfaces, 48 (2006) 176-182.

[12] N. Pyo, S. Tanaka, C.E. McNamee, Y. Kanda, Y. Fukumori, H. Ichikawa, K. Higashitani, Colloids Surf. B: Biointerfaces, 53 (2006) 278-287.

[13] C.E. McNamee, N. Pyo, K. Higashitani, Biophys. J., 91 (2006) 1960-1969.

[14] C.E. McNamee, S. Yamamoto, K. Higashitani, Biophys. J., 93 (2007) 324-334.

[15] C.E. McNamee, Y. Aso, S. Yamamoto, Y. Fukumori, H. Ichikawa, K. Higashitani, Pharm. Res., 24 (2007) 2370-2380. 
[16] H. Shinto, Y. Aso, T. Fukasawa, K. Higashitani, Colloids Surf. B: Biointerfaces, 91 (2012) 114121.

[17] H. Shinto, Y. Ohta, T. Fukasawa, Adv. Powder Technol., (2012).

[18] H. Aoki, Science and Medical Applications of Hydroxyapatite, Japanese Association of Apatite Science, Tokyo, 1991.

[19] P.W. Brown, B. Constantz, Hydroxyapatite and Related Materials, CRC Press, London, 1994.

[20] L.L. Hench, S. Best, in: B.D. Ratner, A.S. Hoffman, F.J. Schoen, J.E. Lemons (Eds.) Biomaterials Science: An Introduction to Materials in Medicine, Elsevier Academic Press, Amsterdam, 2004, Chapter 2.10.

[21] S. Cazalbou, C. Combes, D. Eichert, C. Rey, M.J. Glimcher, J. Bone Miner. Metab., 22 (2004) $310-317$.

[22] T.J. Webster, C. Ergun, R.H. Doremus, R.W. Siegel, R. Bizios, Biomaterials, 22 (2001) 1327-1333.

[23] G. Balasundaram, M. Sato, T.J. Webster, Biomaterials, 27 (2006) 2798-2805.

[24] W. Sun, C. Chu, J. Wang, H. Zhao, J. Mater. Sci.: Mater. Med., 18 (2007) 677-683.

[25] S. Fujii, M. Okada, H. Sawa, T. Furuzono, Y. Nakamura, Langmuir, 25 (2009) 9759-9766.

[26] B. Arechabala, C. Coiffard, P. Rivalland, L.J.M. Coiffard, Y.D. Roeck-Holtzhauer, J. Appl. Toxicol., 19 (1999) 163-165.

[27] J. Wong, A. Brugger, A. Khare, M. Chaubal, P. Papadopoulos, B. Rabinow, J. Kipp, J. Ning, Adv. Drug Delivery Rev., 60 (2008) 939-954.

[28] Y. Mima, S. Fukumoto, H. Koyama, M. Okada, S. Tanaka, T. Shoji, M. Emoto, T. Furuzono, Y. Nishizawa, M. Inaba, PLoS ONE, 7 (2012) e35199.

[29] S. Fujii, M. Okada, T. Furuzono, J. Colloid Interface Sci., 315 (2007) 287-296.

[30] T. Kawasaki, S. Takahashi, K. Ideda, Eur. J. Biochem., 152 (1985) 361-371.

[31] M.S. Ehrenberg, A.E. Friedman, J.N. Finkelstein, G. Oberdörster, J.L. McGrath, Biomaterials, 30 (2009) 603-610.

[32] D. Guarnieri, A. Guaccio, S. Fusco, P.A. Netti, J. Nanoparticle Res., 13 (2011) 4295-4309. 
[33] S. Iyer, R.M. Gaikwad, V. Subba-Rao, C.D. Woodworth, I. Sokolov, Nat. Nanotechnol., 4 (2009) 389-393.

[34] A. Taubenberger, D.A. Cisneros, J. Friedrichs, P.-H. Puech, D.J. Müller, C.M. Franz, Mol. Biol. Cell, 18 (2007) 1634-1644.

[35] R.H. Kramer, M. Vu, Y.-F. Cheng, D.M. Ramos, Cancer Metastasis Rev., 10 (1991) 49-59.

[36] K.M. Woo, J. Seo, R. Zhang, P.X. Ma, Biomaterials, 28 (2007) 2622-2630.

[37] A. Dolatshahi-Pirouz, T. Jensen, M. Foss, J. Chevallier, F. Besenbacher, Langmuir, 25 (2009) 2971-2978. 

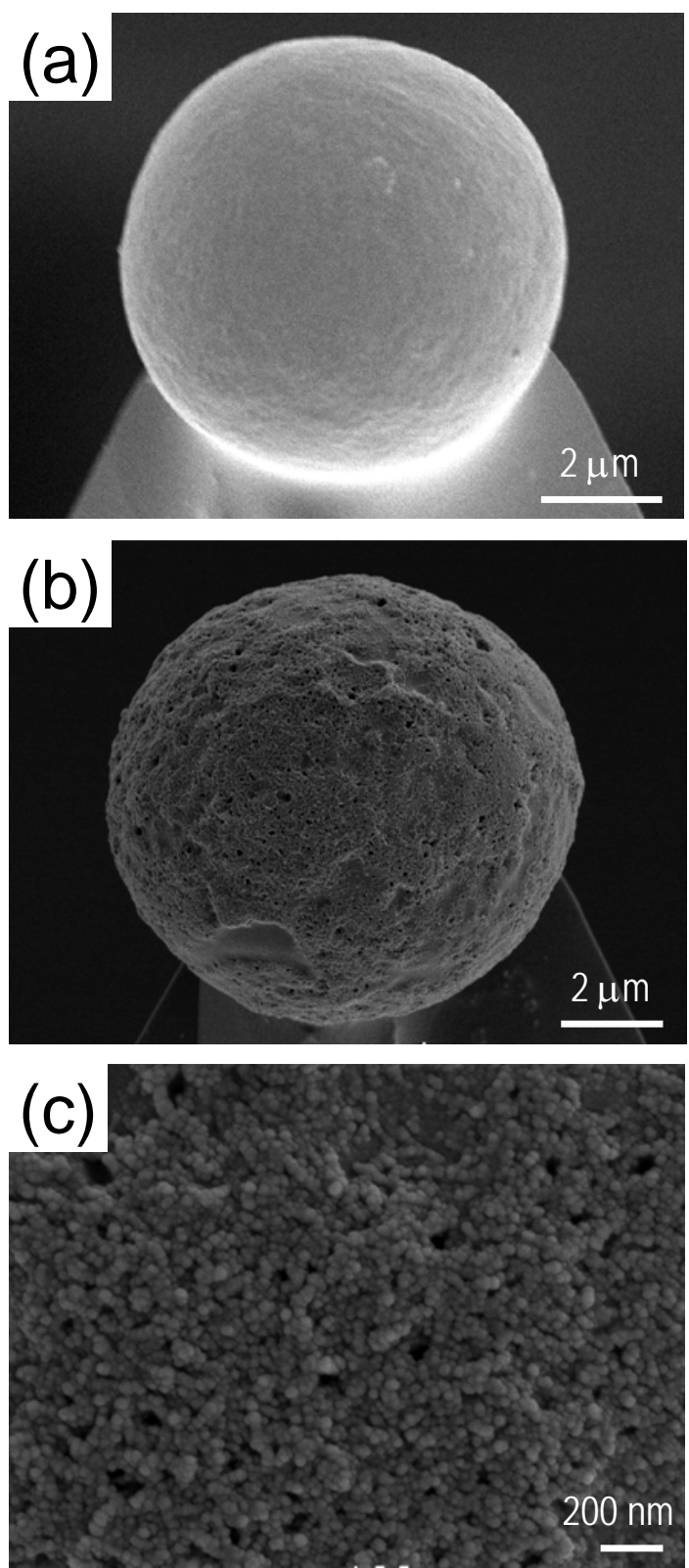

Fig. 1. Shinto et al. 

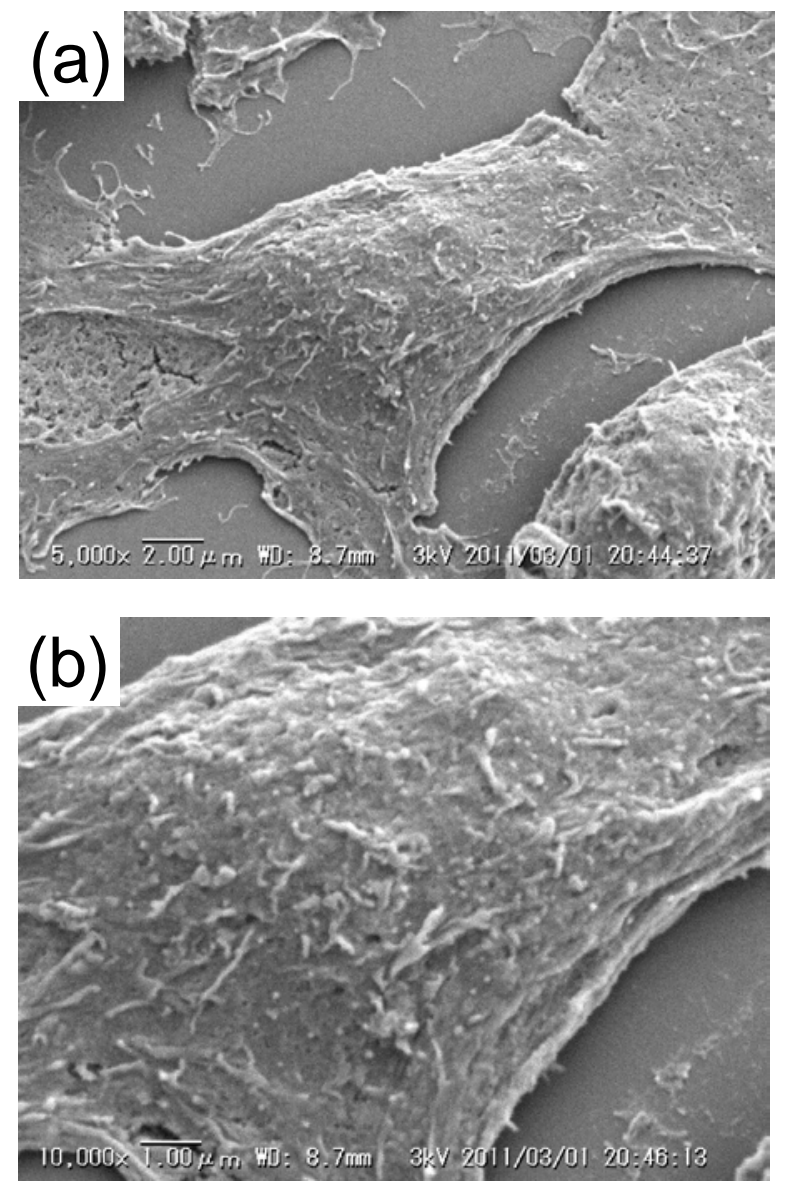

Fig. 2. Shinto et al. 
(a)

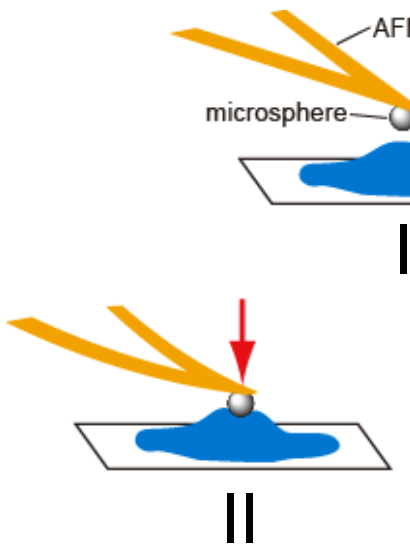

(b)

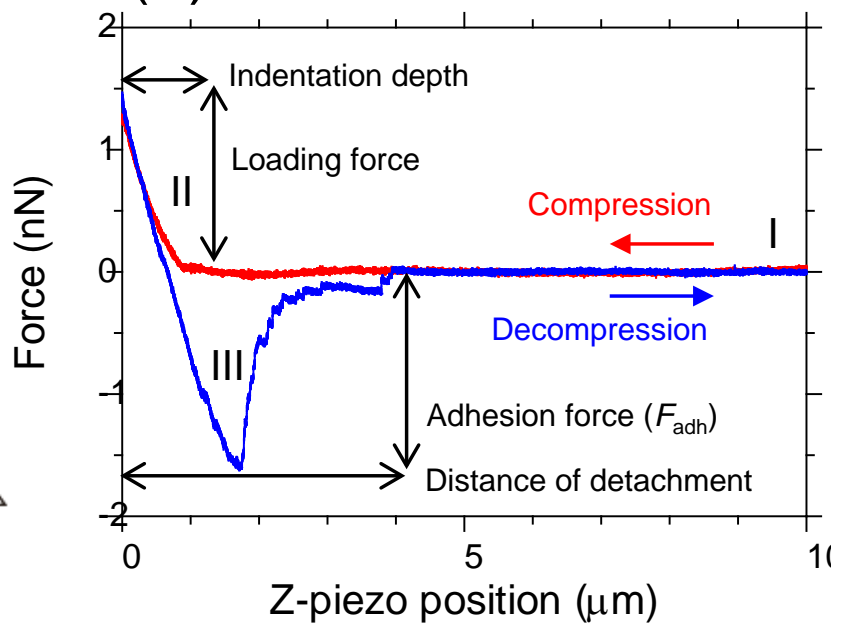

Fig. 3. Shinto et al. 


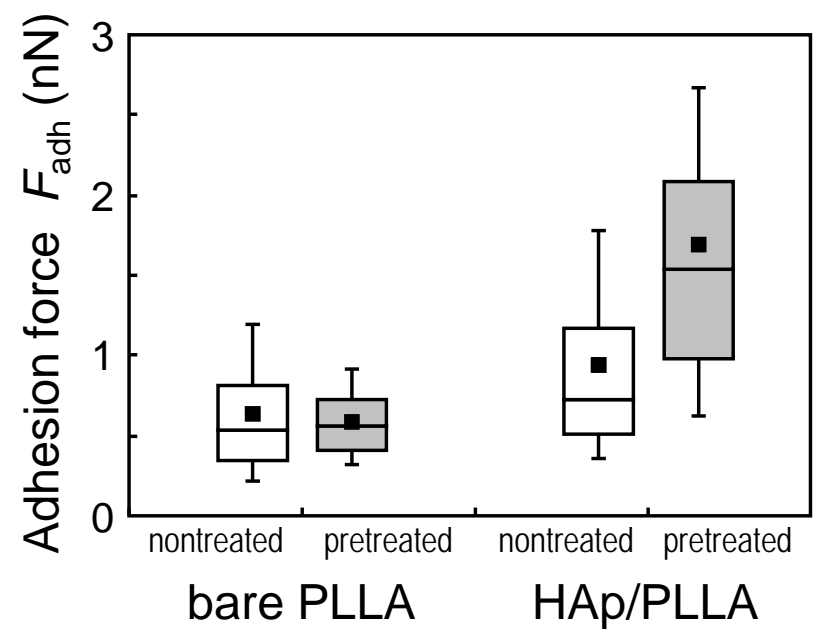

Fig. 4. Shinto et al. 


\section{Electronic Supplementary Data}

\section{Effect of interfacial serum proteins on melanoma cell adhesion to biodegradable poly(L-lactic acid) microspheres coated with hydroxyapatite}

Hiroyuki Shinto, ${ }^{*}, 1$ Takuya Hirata, ${ }^{1}$ Tomonori Fukasawa, ${ }^{1}$ Syuji Fujii, ${ }^{2}$ Hayata Maeda, ${ }^{2}$ Masahiro Okada, ${ }^{3}$ Yoshinobu Nakamura, ${ }^{2}$ and Tsutomu Furuzono ${ }^{4}$

${ }^{1}$ Department of Chemical Engineering, Kyoto University, Katsura, Nishikyo-ku, Kyoto 615-8510, Japan

2 Department of Applied Chemistry, Faculty of Engineering, Osaka Institute of Technology, 5-16-1 Ohmiya, Asahi-ku, Osaka 535-8585, Japan

${ }^{3}$ Department of Biomaterials, Osaka Dental University, 8-1 Kuzuha-Hanazono, Hirakata, Osaka5731121, Japan

${ }^{4}$ Department of Biomedical Engineering, School of Biology-Oriented Science and Technology, Kinki University, 930 Nishi-Mitani, Kinokawa, Wakayama 649-6493, Japan

\footnotetext{
* Author to whom correspondence should be addressed.

Phone: +81-75-383-2671, Fax: +81-75-383-2651, e-mail: shinto@cheme.kyoto-u.ac.jp
} 
(a) bare PLLA, nontreated

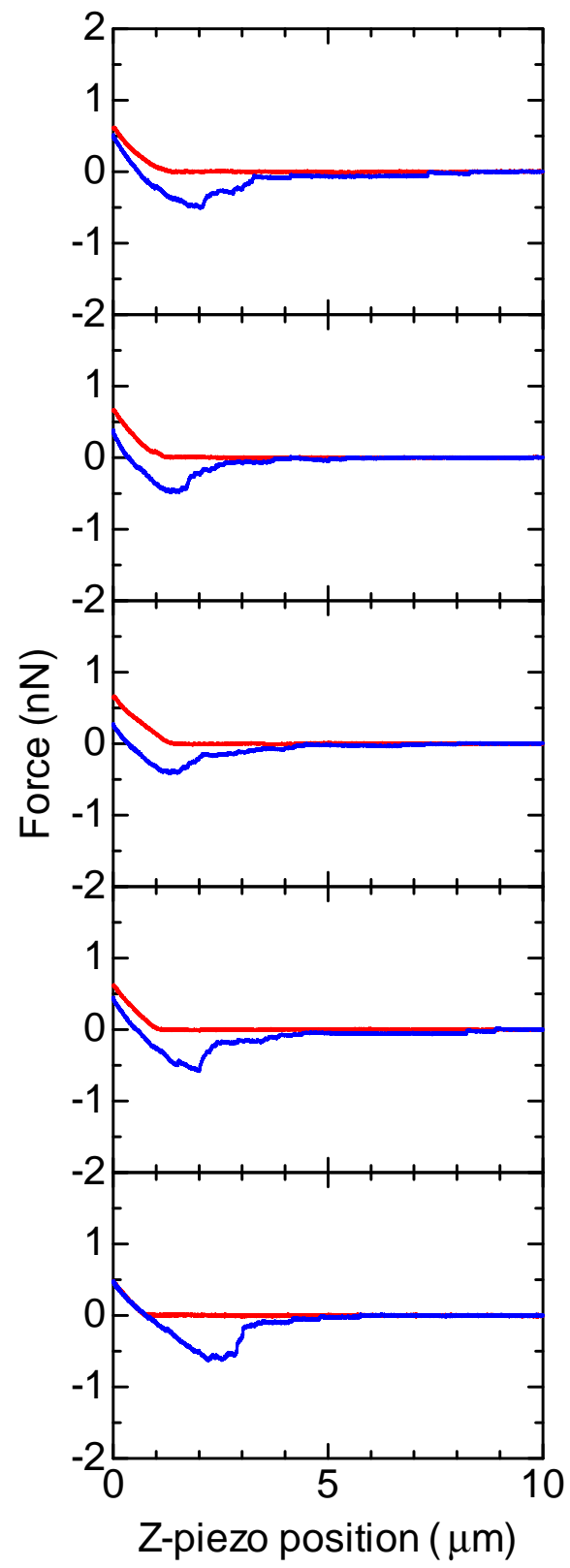

(b) bare PLLA, pretreated

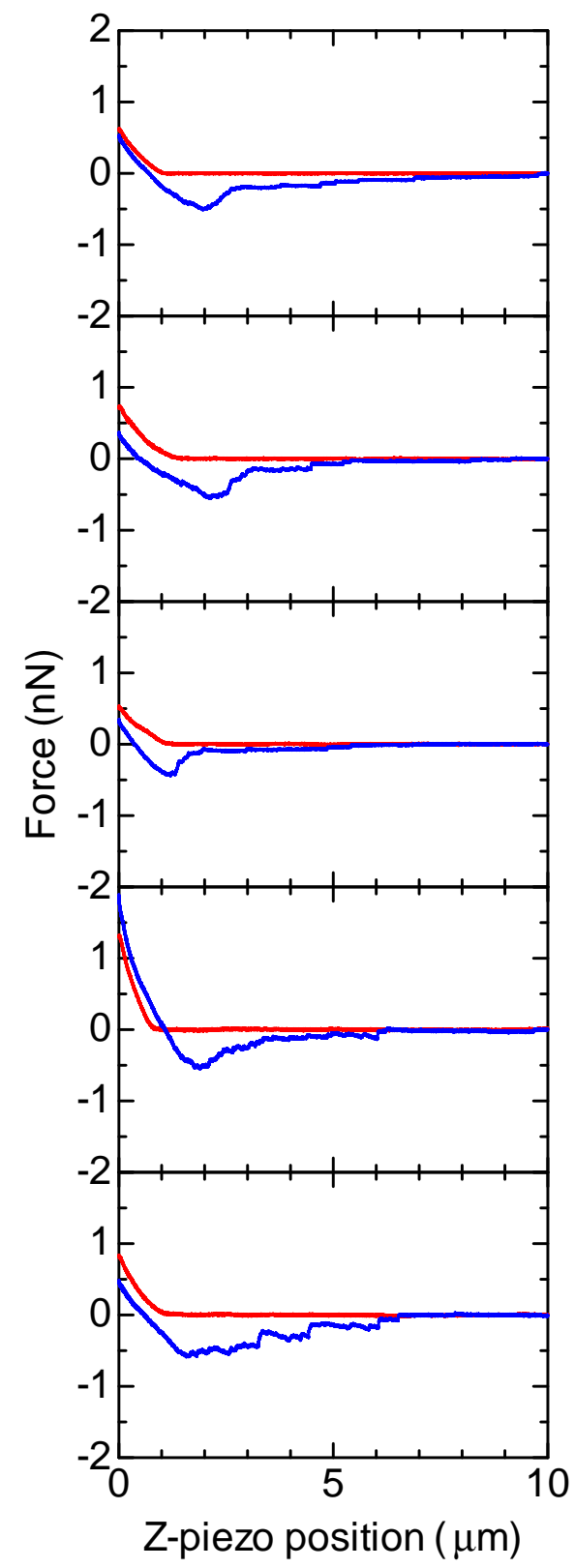

Fig. S1. Representatives of force-displacement curves during compression (red colored) and decompression (blue colored) measured between a B16F10 cell and a bare PLLA microsphere of 8-10 $\mu \mathrm{m}$ diameter in a serum-free culture medium of DMEM: (a) the nontreated microspheres and (b) the microspheres pretreated with a 10\% FBS-supplemented DMEM. The dwell time, indentation depth, and Z-scan speed were $5 \mathrm{~min}, 1.3 \pm 0.3 \mu \mathrm{m}$, and $0.8 \mu \mathrm{m} / \mathrm{s}$, respectively. 
(a) HAp/PLLA, nontreated

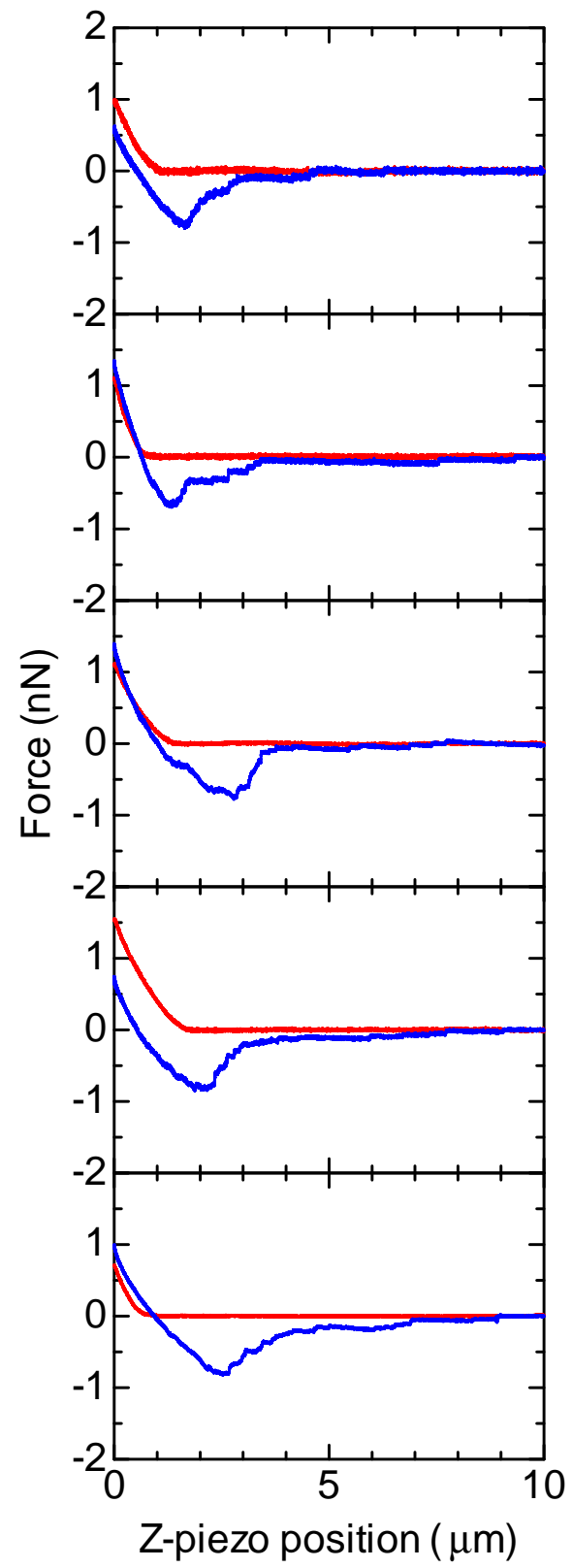

(b) HAp/PLLA, pretreated

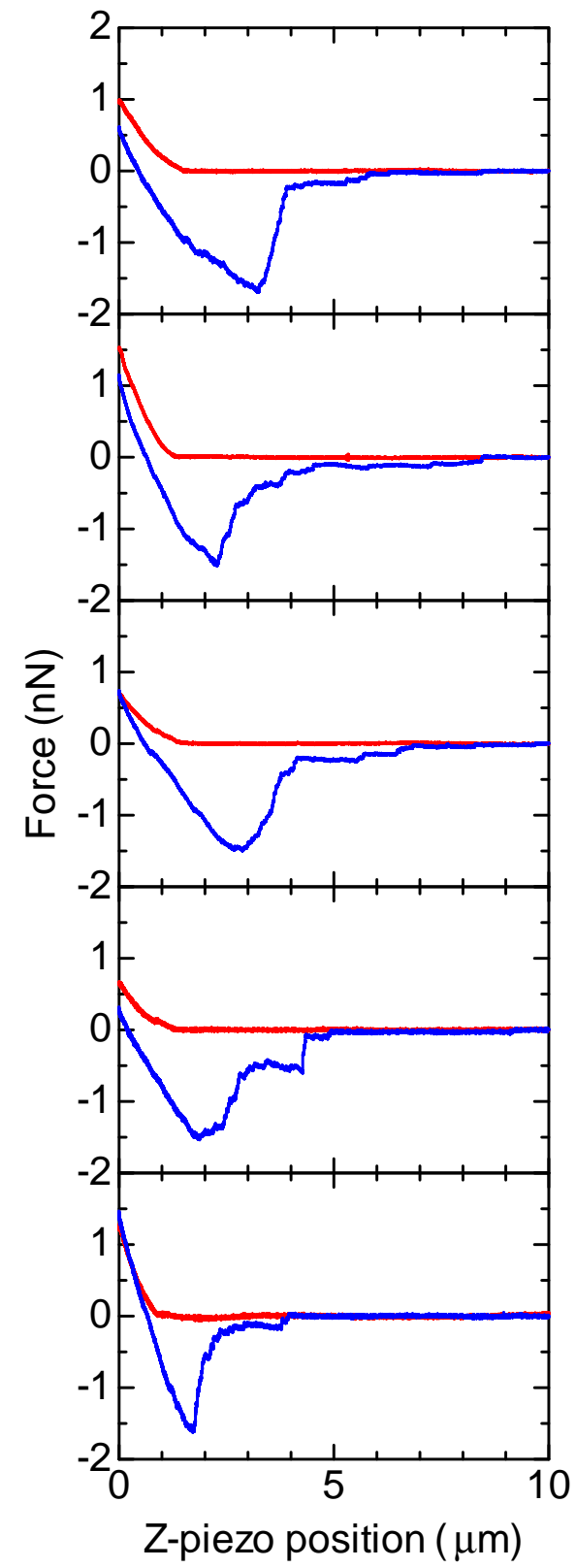

Fig. S2. Representatives of force-displacement curves during compression (red colored) and decompression (blue colored) measured between a B16F10 cell and an HAp/PLLA microsphere of 8-10 $\mu \mathrm{m}$ diameter in a serum-free culture medium of DMEM: (a) the nontreated microspheres and (b) the microspheres pretreated with a 10\% FBS-supplemented DMEM. The dwell time, indentation depth, and Z-scan speed were $5 \mathrm{~min}, 1.3 \pm 0.3 \mu \mathrm{m}$, and $0.8 \mu \mathrm{m} / \mathrm{s}$, respectively. 